\title{
Constructive Processes in Completing Reading-to-Write Tasks: Selecting, Organising and Connecting
}

\author{
Pucheng Wang, Zuogong Zhang* \\ School of Applied Foreign Languages, Zhejiang International Studies University, Hangzhou, China \\ Email: p.wang@zisu.edu.cn, ^timenb@126.com
}

How to cite this paper: Wang, P. C., \& Zhang, Z. G. (2021). Constructive Processes in Completing Reading-to-Write Tasks: Selecting, Organising and Connecting. Open Journal of Modern Linguistics, 11, 919-930. https://doi.org/10.4236/ojml.2021.116071

Received: November 30, 2021

Accepted: December 14, 2021

Published: December 17, 2021

Copyright (๑) 2021 by author(s) and Scientific Research Publishing Inc. This work is licensed under the Creative Commons Attribution-NonCommercial License (CC BY-NC 4.0).

http://creativecommons.org/licenses/by-nc/4.0/

\begin{abstract}
This study investigated how 16 English as a Foreign Language writers constructed meaning from texts through reading and for texts through writing in responding to a source-based writing task. Results showed that most of the writers engaged in selecting, organising and connecting processes during task completion. The participants not only selected information from source materials, but they also searched for ideas from their prior knowledge in order to generate links or new meaning. Organising processes were found before the participants started to write, although they were more prone to construct the structure of the source materials and their own text concurrently during writing. Evidence from the participants' eye movements and verbal recalls proved that connecting and generating are not a one-off act, but an extended and ongoing process that may occur at different phase of reading and writing.
\end{abstract}

\section{Keywords}

Second Language Writing Assessment, Integrated Writing Tasks, Constructive Processes, Discourse Synthesis

\section{Introduction}

Reading-to-write tasks engage writers with both reading and writing skills. Over the past four decades, a significant amount of research has investigated writers' cognitive processes involved in completing reading-to-write tasks (Gebril \& Plakans, 2013; Golparvar \& Khafi, 2021; Shi, 2004; Spivey, 1984, 1997; Wang et al., 2020; Weigle \& Parker, 2012). One important notion that emerged from these studies is the concept of discourse synthesis. It is a constructive process during which writers transform a new representation of the meaning from source 
materials to their own text (Mathison \& Spivey, 1993; Spivey, 1990, 2001; Spivey \& King, 1989). Three sub-processes are involved in it: 1) selecting relevant ideas or information from source materials; 2) organising ideas as writers read and write; and 3) connecting ideas selected from different source materials and generate links between them.

Discourse synthesis is arguably the most unique and essential process in completing reading-to-write tasks. Compared with the abundance of studies on this process in L1 writing research, however, there has been relatively less research on it in the literature of L2 writing. Among the studies that attempted to investigate L2 writers' discourse synthesis process, most of them have looked at the process through examining writers' written products, few studies looked at writers' online constructive processes. Therefore, a need exists for the field to improve our understanding of how L2 or EFL writers construct meaning as they read and write to inform language teachers and learners the nature of this process and facilitate the teaching and learning of it. The present study investigated 16 EFL writers' constructive processes while completing a source-based readingto-write task, aiming to gain further insight into writers' cognitive processing related to selecting, organising and connecting in an EFL context.

\section{Studies on Constructive Processes}

\subsection{Selecting Process}

As mentioned earlier, selecting is a process used when writers select relevant ideas or information from the source materials or their long-term memory to put into the text they are going to produce. Spivey (1991) argued that selecting plays an important role in meaning construction because the new meaning constructed is based on the ideas writers select from either internal or external sources.

Scardamalia and Bereiter (1987) found that advanced and immature writers employed selecting processes very differently. Immature writers select ideas simply by ranking information according to importance when recalling knowledge from memory, while advanced writers devote more cognitive effort in selecting content by resorting to a set of criteria, for example, the relevance to the writing goals, the appropriateness for intended readers, and fitness to the overall structure.

\subsection{Organising Process}

In a traditional independent writing task, organising is a process in which writers organise the ideas to be put into their text by evaluating their priorities and relevance to topic of the task, while in an integrated reading-to-write task, as Spivey (1991) argued, writers not only order ideas in their own text, but they also organise the relationships between ideas in the source texts to achieve an understanding of the text.

Scardamalia and Bereiter (1987) discovered that writers who adopted a know- 
ledge-telling approach to writing devoted little effort in ordering the ideas to be put in the text, but generated text in a rather linear fashion, that is, put down ideas in the order as they were retrieved from the long-term memory. This is like dumping all the relevant knowledge in writers' mind at once, a process often found in writing-disabled students whose ability to plan is believed to be disrupted (Cherkes-Julkowski, Sharp, \& Stolzenberg, 1997). On the other hand, writers who adopted a knowledge-transforming approach to writing were actively engaged in organising process as they transformed the ideas from their mind into the text by ordering and prioritising these ideas based on the evaluation of their relevance and importance to the writing goals.

\subsection{Connecting Process}

Connecting is a process in which writers bring what they already know into the reading and create meaning-enhancing additions (Levin, 1988). In other words, writers combine the knowledge they retrieve from memory with the ideas they select from source materials, and generate either links between these ideas or new meaning (Spivey, 1987). As they select and connect during reading, they are creating a pool of ideas from which to draw for the writing process (Stein, 1990). The output of selecting and connecting may ultimately become the basis of plans for the writing.

As discussed in Bereiter and Scardamalia's (1987) model, writers who adopt the knowledge-telling approach engage in a rather linear and straightforward writing process, during which the main activities are retrieving ideas from memory and putting them into the text. They are less likely to connect ideas in the source texts with their own knowledge when writing from sources. In contrast, skilled writers who use a knowledge-transforming approach tend to constantly connect ideas from their memory and source texts to generate ideas for the new text. These new ideas may be repetitive and vary in importance to the writing goals, thus the process of organising may be activated to address these issues.

Chan (2013) used a self-developed reading-to-write process questionnaire to investigate 219 students' cognitive processes while completing four reading-to-write tasks under real-life and test conditions. The results of exploratory factor analysis confirmed the underlying construct of different cognitive processes that Chan proposed as core processes in a reading-to-write task. Her findings also revealed that higher-scoring students reported more use of connecting processes than lower-scoring students did.

\subsection{Research Questions}

The complex processes involved in writers' meaning construction in readingwriting have been examined through a variety of methodological approaches in previous studies. However, these approaches (for example, think-aloud protocols, questionnaire) have different drawbacks when used alone, and are liable to 
over-generalise the findings beyond the limitation of each method.

Therefore, in order to address this methodological issue, this study combined eye-tracking technique and stimulated recall method to look into EFL writers' constructive processes while completing a reading-to-write task, hoping to triangulate data from different sources, and thus enhancing the validity of the research findings. Three research questions were proposed:

1) How do EFL writers select internal and external ideas/information as they read and write? (RQ1)

2) How do EFL writers organise ideas/information as they read and write? (RQ2)

3) How do EFL writers connect ideas/information and generate links between them or new meaning as they read and write. (RQ3)

\section{Method}

\subsection{Participants}

A total of 16 university students participated in this study. They were all native Chinese learners of English studying at a university in the UK. Eleven of them were female (69\%) and five were male (31\%); their ages were between 21 and 28 years $($ Mode $=23$; Mean $=22.6$; $\mathrm{SD}=1.66$ ). All the participants had sat the International English Language Testing System (IELTS) test before data collection. Their scores were presented in Table 1. According to the Common European Framework of Reference for Languages (CEFR), these participants' English proficiency levels ranged from $\mathrm{B} 2$ to $\mathrm{C} 1$.

\subsection{Instrument and Data Collection}

A Tobii TX300 eye-tracker was used to record the participants' eye movements as they respond to the stimulus onscreen, which is a sample reading-to-write task of the Test for Business English Majors-Band 8 (developed and administered in China). The task prompt consists of an instruction and five source materials about Steve Jobs' resignation on Apple. During the experiment, the task was displayed on the eye-tracker screen: the instruction and three source texts were presented down the left part of the screen, and the other two source materials plus the answer sheet box (where the participants input the text) were presented on the right part of the screen.

Table 1. Participants' IELTS test scores.

\begin{tabular}{ccccccc}
\hline $\begin{array}{c}\text { IELTS/IELTS } \\
\text { components }\end{array}$ & Mean & Median & Mode & $\begin{array}{c}\text { Standard } \\
\text { Deviation }\end{array}$ & Minimum & Maximum \\
\hline Overall & 7.16 & 7.00 & 7.50 & 0.35 & 6.50 & 7.50 \\
Reading & 8.00 & 8.00 & 8.50 & 0.58 & 7.00 & 9.00 \\
Writing & 6.25 & 6.00 & 6.00 & 0.55 & 5.50 & 7.00 \\
\hline
\end{tabular}


During the data collection, the participants were first responding to the task with their eye movements being recorded by the eye-tracker. When they finished the task, they were then asked to verbalise their thoughts during task completion, using their eye traces recorded by the eye-tracker as stimuli for retrospection. The stimulated recall session was conducted in Mandarin Chinese, and was audio and video recorded for further data analysis.

\subsection{Data Analyses}

In order to answer the three research questions, the participants' verbal recalls were first transcribed by one of the researchers (a native Chinese speaker) based on the audio and video recordings of the stimulated recall session. Then the transcriptions were coded to identify students' constructive processes (selecting, organising and connecting processes) throughout the task completion, and the number of occurrences for each type of constructive processes at different stages of task completion was calculated. In addition, quotes from participants' verbal protocols were presented to illustrate their use of constructive processes while completing the reading-to-write task.

\section{Results and Discussion}

\subsection{Selecting Process (RQ1)}

A total of 307 instances of selecting process were found in the protocols. They mainly served three purposes: 1) to select ideas from both memory and source materials for connecting; 2) to select information (often at the level of detail, for example, a specific word) from source materials to support writing; 3 ) to select sentences from source materials for paraphrasing or translating.

First, when the participants composed from sources, they selected ideas from both their prior knowledge and source materials (91 instances), connecting them to generate either links between ideas or new meaning. By using this type of selecting, together with the process of connecting and generating, participants created a pool of ideas, from which they would like to draw during writing.

Another type of selecting, which was more frequently reported by the participants in their stimulated recalls, was to select specific information from the source materials to support the writing process (182 instances). This information was, most of the time, a certain word that the participants decided to bring into their own writing. For example, Participant 1, while writing a sentence in the second paragraph of her essay, said, "I was thinking about what noun I can use to describe him (Steve Jobs), I found the word 'leadership' above, so I decided to use leader". Similarly, Participant 3 explained why he went to look at the words in the "key concepts and expressions" box in the task prompt, "Then I went on writing, I wanted to talk about how the Apple company kept developing, I was wondering if there was any word in the 'key words' list that can be used in my writing, and then I found the phrase 'differential competitive advantage', so I added it into my essay". 
These two examples indicate that the words in the source materials provided some lexical support for the participants' composing process, especially when they were trying to transcribe their abstract ideas into concrete linguistic forms. The participants also reported that they sometimes went back to the materials during writing to look for words that they could not recall correctly after their reading of the sources, for example, Participant 5 stated that " $I$ went back to the source material to find the word 'resign', I wanted to make sure whether there is an ' $s$ ' or two 'ss' in it', and "I wanted to use the word 'charisma', so I went back to find it".

Last, searching for specific information and selecting it from the source materials was another common activity performed by these participants, when they found that certain information they read before was needed in their writing, and they would go back to the materials to look for it, for instance, Participant 8 , when writing a sentence introducing the Apple products designed by Steve Jobs, said, "I was going to write some examples, what products had been designed, so I went back to read the first paragraph".

The third type of selecting process (34 instances) discovered in the 16 participants' protocols was to select, paraphrase and/or translate original sentences in the source materials and integrated them into the participants' own writing. Fourteen participants reported that they engaged in this type of selecting during writing. It seemed that the participants were aware of the restraints in the instructions that they "should not simply copy and translate the source materials", so they adopted paraphrasing as a means of incorporating the sentences in the materials, for example, Participant 9 said, "This sentence was to introduce what I was going to write in my essay. Basically, I paraphrased the task requirements in the instructions, so I would look at the instructions" and:

I was looking for the information about his post, although the instructions said that "you should not copy", I was basically paraphrasing that sentence... so in this paragraph, I was composing sentences and looking at that source material at the same time. That's why my eye fixations frequently switched between these two areas.

Also, as the input materials included Chinese texts, the participants were found simply translating sentences in these materials and put them into their own writing, for instance, Participant 5 stated that "I used the information in the third source material. I was translating those Chinese sentences". Participant 13 even claimed that she copied one sentence in the materials, "I was writing about its management team, and I just copied the sentence talking about that".

In summary, when the participants composed from sources, there was ample evidence to support the notion that they used the selecting process to choose ideas from both memory and source materials, then connected them to generate new meaning that may have value for the content of their writing. Also, these participants were found, more frequently, to seek both lexical and syntactical support from the source materials during the process of translating (transcribing abstract ideas into linguistic forms), which concurs with the findings in their 
eye-traces, that they constantly switched their attention between different parts of the task during writing.

\subsection{Organising Process (RQ2)}

During the organising process, writers were either organising the relationships between ideas in the source materials and/or structuring their own writing. It was hypothesised that writers may have difficulty in generating coherent texts if they could not first assemble the texts they read into a unified coherent whole; in other words, they would need to build a representation of source materials in order to build a representation of their own text (Stein, 1990).

The 16 participants' protocols were parsed to differentiate the use of the two types of organising process between different writing phases. The number of occurrences for each type of organising process is displayed in Table 2 and Table 3. As shown in the tables, a total of 60 instances of using organising process were found in the participants' verbal recalls. Both before and during writing, the participants spent time organising (42 instances) to support their reading. Before writing (25 instances), they not only tried to comprehend the ideas in the source materials, but they also tried to discover the relationships between these ideas for the text they were about to produce. For example, Participant 2 recalled her organising process before starting to write:

I was reading through the source materials, there were some relationships between them, some of them were talking about the same issue... there were two points of view in these materials, one was that Apple Company would not change after Jobs' resignation, the other one was that his resignation would have impact on the company, I categorised these materials into the two sides.

Table 2. Organising-thinking about the structure of the source materials at different phase of writing by participant.

\begin{tabular}{|c|c|c|c|c|c|c|c|c|c|c|c|c|c|c|c|c|c|}
\hline \multirow{2}{*}{ Writing phase } & \multicolumn{16}{|c|}{ Participant } & \multirow[b]{2}{*}{ Total } \\
\hline & 1 & 2 & 3 & 4 & 5 & 6 & 7 & 8 & 9 & 10 & 11 & 12 & 13 & 14 & 15 & 16 & \\
\hline Before writing & 3 & 4 & 4 & 0 & 2 & 5 & 0 & 1 & 0 & 1 & 3 & 0 & 1 & 0 & 0 & 1 & 25 \\
\hline During writing & 4 & 3 & 0 & 2 & 1 & 0 & 0 & 1 & 1 & 2 & 0 & 0 & 2 & 1 & 0 & 0 & 17 \\
\hline After writing & 0 & 0 & 0 & 0 & 0 & 0 & 0 & 0 & 0 & 0 & 0 & 0 & 0 & 0 & 0 & 0 & 0 \\
\hline Total & 7 & 7 & 4 & 2 & 3 & 5 & 0 & 2 & 1 & 3 & 3 & 0 & 3 & 1 & 0 & 1 & 42 \\
\hline
\end{tabular}

Table 3. Organising-thinking about the structure of their writing at different phase of writing by participant.

\begin{tabular}{ccccccccccccccccccc}
\hline \multirow{2}{*}{ Writing phase } & \multicolumn{11}{c}{ Participant } \\
\cline { 2 - 5 } & 1 & 3 & 4 & 5 & 6 & 7 & 8 & 9 & 10 & 11 & 12 & 13 & 14 & 15 & 16 & Total \\
\hline Before writing & 0 & 0 & 0 & 1 & 0 & 1 & 0 & 0 & 0 & 0 & 0 & 0 & 0 & 0 & 0 & 0 & 2 \\
During writing & 3 & 0 & 1 & 0 & 0 & 1 & 0 & 0 & 1 & 1 & 3 & 1 & 0 & 2 & 0 & 3 & 16 \\
After writing & 0 & 0 & 0 & 0 & 0 & 0 & 0 & 0 & 0 & 0 & 0 & 0 & 0 & 0 & 0 & 0 & 0 \\
$\quad$ Total & 3 & 0 & 1 & 1 & 0 & 2 & 0 & 0 & 1 & 1 & 3 & 1 & 0 & 2 & 0 & 3 & 18 \\
\hline
\end{tabular}


Over half of the participants reported that they also spent time trying to understand the source materials during writing (17 instances), using different strategies such as summarising the main ideas for each reading and identifying rhetorical structures (Plakans, 2009). For instance, Participant 2 said:

I was looking for descriptions of Jobs, what kind of person he was, how talented he was, I was looking for materials about Jobs, the first source material described the Apple Company, so I did not look at it, the other two source texts talked about Jobs. I went to the third material to look for descriptions of Jobs and comments on him.

And Participant 10 stated, "I was reading these two paragraphs (in the third source material), because they seemed to talk about the same issues, so I kept reading them back and forth and tried to summarise main ideas from them". No instances of the organising process were found after the participants finished writing the first draft.

Eighteen instances were devoted to organising the ideas to be put in the essays (see Table 3). It is worth noting that only two participants (Participants 4 and 6) reported in their protocols that they were thinking about the overall structure of their writing before they started to produce any text. For example, Participant 6 said, "I was reading (the source materials) and thinking about how to write the introduction part, the transition paragraphs, and the ending paragraph". Most of the instances of structuring the essays were found during writing, when participants either referred back to the source materials to build a clearer representation of the input texts and then continued to think about the structure of their own writing in order to incorporate different ideas from the materials, or they just went about structuring the essays based on their own evaluations, for example, Participant 16 stated, "I thought these two paragraphs were both descriptive ones, it's not necessary to separate them, so I put them together, and then I decided to start a new paragraph and to analyse the situation".

In summary, the participants did appear to spend time thinking about the structure of the source materials while completing the reading-to-write task, especially before they started to produce any text, however few of them had worked out a rough outline of key points to guide their writing, instead, most participants seemed more prone to using strategies to understand the source materials during writing, while concurrently structuring the text to be produced. This is likely due to the complexity and difficulty of the organising process in completing an integrated writing task which includes source materials; writers may not be able to create a complete representation of the input texts instantly, which impedes the progress of building their own text on the source materials.

\subsection{Connecting Process (RQ3)}

Connecting is a process in which writers bring what they already know into the reading and create meaning-enhancing additions (Levin, 1988). In other words, writers combine their prior knowledge with the ideas they select from source 
materials to generate new meaning. As they connect and generate during the reading phase, they are "creating a pool of ideas from which to draw during the writing process" (Stein, 1990: p. 147). To some extent, then, the selecting, organising and connecting of ideas that occurs during reading may become the basis of plans for their writing.

The 16 participants reported 91 instances of connecting and generating through task completion. Most of these instances occurred during writing; few participants reported that they connected ideas in the source materials with their own knowledge while reading the materials before writing. This may be because of the limitation of the stimulated recall methodology that participants may not be able to recollect adequately their thought processes at the beginning of task completion due to memory decay. On the other hand, it may also be the case that the participants did actually not engage in the connecting process before writing, instead, they were prone to selecting, connecting and organising ideas from both the source materials and/or their memory during writing.

Analysis of the 16 participants' protocols indicates that the use of connecting processes during writing mainly served two different purposes: 1) to develop ideas already found in the source materials; 2) to generate new ideas. First, almost all the participants reported that they used connecting as a means of elaborating ideas found in the source texts (73 instances), when they selected relevant ideas from the input texts, combined them with their prior knowledge, and generated further development of these ideas. For example, Participant 1, when writing the second paragraph of her essay, said:

I went to read some sentences that I had written in this paragraph, and was probably thinking about what to write for the next sentence. I found the "market value" (in the word list) above, I thought I could write on this, and also I could write something about the "advantage" above...

And this elaboration of ideas from the source texts became what she wrote in her final essay, that is:

the world will not be surprised to see this listed company suffer from a decreased market value without much differential competitive advantage over its counterparts any more.

The other function of connecting is to produce different kinds of additional materials, i.e., information not found in the source materials, much of which is at a very detailed level, for example, new words that participants include in their essays. In this analysis, however, the generation of new materials refers specifically to those instances when the participants were attempting to re-evaluate the ideas provided in the source materials, by connecting their own knowledge with them, and generated ideas from a new perspective. Thus, this function of connecting requires writers to elaborate more critically on the ideas found in the source texts. Six of the 16 participants recalled in their protocols that they engaged in this type of connecting and generating process (18 instances). The protocol of Participant 11 provides some good examples of it. For example, when 
reading the third source material before starting to write, she said:

when I was reading this material, I was thinking (about the topic), it says that the Jobs' resignation on Apple had no great impact on the development of the company, but in my point of view, considering the current status of Apple, there is great impact...so when I was reading this material, I was also thinking about if there were any counter examples that could prove that his resignation did impact on the development of Apple.

This generation of new ideas during reading also became part of this participant's writing plan and content later when she was writing the third paragraph of her essay, where she wrote:

"Jobs' leave can be a severe loss of Apple" and stated the reason: "since Jobs' personal charisma is a very significant identity icon of Apple and maybe even the power gathering all the talents together".

In summary, there is evidence of the usefulness of the connecting process in the 16 participants' stimulated recalls. It may lead to elaboration of the ideas found in the source materials, by applying the participants' prior knowledge (including world knowledge, experiences, preferences etc.) to what they were reading, and may also promote critical thinking, creating ideas from a new perspective, when participants used their prior knowledge as a basis for comparison and evaluated the validity of the propositions in the source materials. Eventually, the process of connecting creates an individualised pool of ideas; as the participants planned their writing, and made decisions about what to write, they would select information from that pool. The protocols also show that most of the participants used connecting process during writing, when they constantly referred back to the source materials (this can also be demonstrated by the eye-tracking data) when producing text for their writing; this may be likely due to the difficulty of building a complete representation of these source materials at the start of task completion, and the fact that the connecting and generating is an ongoing process of meaning building, rather than a one-off act. Participant 10 provided an explanation on this:

... at the beginning, I first read through the materials, and generated an overall impression, which might not be completely clear, but I knew roughly what it was in different part of the essay I should write, then during writing, when I felt that some information might be missed, I would go back to the materials to check if there were any content worth adding into the writing.

As the participants went on reading and writing, their representations of the source materials and the essay both became clearer and more complete, and then a coherent written product was more likely to be produced.

\section{Conclusion}

This study examined EFL writers' constructive processes while completing a source-based reading-to-write task. Findings from the participants' verbal protocols reveal that, as they read and write to complete the task, most of them: 1) 
used selecting processes to choose ideas from either memory or source materials in order to construct new meaning, and they were found frequently seeking lexical and syntactical support from the source materials; 2) spent time to think about the structure of the source materials before writing although few participants worked out a plan for the text to be produced, and they were more prone to construct the structure of the source materials and their own writing roughly at the same time; 3) connected information or ideas they found in the source materials with their prior knowledge, elaborating these ideas and eventually created an individualised pool of ideas for them to choose from.

The major outcome for this study increased insight into how EFL writers construct meaning from texts through reading and for texts through writing (Spivey, 1990), with which EFL teachers may better plan their lessons to teach these implicit but essential processes for reading and writing. For example, they could develop practising tasks that focus on strengthening the use of the three constructive processes examined in this study to increase students' awareness of discourse synthesis process in reading-writing. Also, EFL learners could be guided by the results of this study to have a clearer understanding of how they could effectively use selecting, organising and connecting processes in responding to reading-to-write tasks in both classroom and testing settings.

One limitation of the study is that the combined use of eye-tracking and stimulated recall methods is quite time-consuming and labour-intensive, and it could be only applied to a relatively small number of students, so that interpreting the results too broadly would pose risks, and any conclusions drawn should be seen as tentative. This limitation, in future research, could be offset by concurrent use of instruments (for example, questionnaire) which can gather data from a larger number of participants, thus triangulating data from different sources and increasing the representativeness of the results.

\section{Conflicts of Interest}

The authors declare no conflicts of interest regarding the publication of this paper.

\section{References}

Bereiter, C., \& Scardamalia, M. (1987). The Psychology of Written Composition. Hillside, NJ: Lawrence Erbaum Associates.

Chan, S. H. C. (2013). Establishing the Validity of Reading-Into-Writing Test Tasks for the UK Academic Context. Unpublished PhD Thesis, The University of Bedfordshire.

Cherkes-Julkowski, M., Sharp, S., \& Stolzenberg, J. (1997). Rethinking Attention Deficit Disorders. Brookline Books.

Gebril, A., \& Plakans, L. (2013). Toward a Transparent Construct of Reading-To-Write Tasks: The Interface between Discourse Features and Proficiency. Language Assessment Quarterly, 10, 9-27. https://doi.org/10.1080/15434303.2011.642040

Golparvar, S. E., \& Khafi, A. (2021) The Role of L2 Writing Self-Efficacy in Integrated Writing Strategy Use and Performance. Assessing Writing, 47, 1-15. 
https://doi.org/10.1016/j.asw.2020.100504

Levin, J. R. (1988). Elaboration-Based Learning Strategies: Powerful Theory = Powerful Application. Contemporary Educational Psychology, 13, 191-205. https://doi.org/10.1016/0361-476X(88)90020-3

Mathison, M. A., \& Spivey, N. N. (1993). Writing from Academic Sources: Authorship in Writing the Critique. National Center for the Study of Writing and Literacy.

Plakans, L. (2009). Discourse Synthesis in Integrated Second Language Writing Assessment. Language Testing, 26, 561-587. https://doi.org/10.1177/0265532209340192

Scardamalia, M., \& Bereiter, C. (1987). Knowledge Telling and Knowledge Transforming in Written Composition. In S. Rosenberg (Eds.), Advances in Applied Psycholinguistics, Volume 2: Reading, Writing and Language Learning (pp. 142-175). Cambridge University Press.

Shi, L. (2004). Textual Borrowing in Second-Language Writing. Written Communication, 21, 171-200. https://doi.org/10.1177/0741088303262846

Spivey, N. N. (1984). Discourse Synthesis: Constructing Texts in Reading and Writing. Outstanding Dissertation Monograph, International Reading Association.

Spivey, N. N. (1987). Construing Constructivism: Reading Research in the United States. Poetics, 16, 169-192.

Spivey, N. N. (1990). Transforming Texts: Constructive Processes in Reading and Writing. Written Communication, 7, 256-287. https://doi.org/10.1177/0741088390007002004

Spivey, N. N. (1991). The Shaping of Meaning: Options in Writing the Comparison. Research in the Teaching of English, 25, 390-418. https://www.jstor.org/stable/40171203

Spivey, N. N. (1997). The Constructivist Metaphor: Reading, Writing and the Making of Meaning. Academic Press. https://doi.org/10.2307/358470

Spivey, N. N. (2001). Discourse Synthesis: Process and Product. In Discourse Synthesis: Studies in Historical and Contemporary Social Epistemology (pp. 379-396). Praeger.

Spivey, N., \& King, J. R. (1989). Readers as Writers Composing from Sources. Reading Research Quarterly, 24, 7-26. https://www.jstor.org/stable/748008 https://doi.org/10.1598/RRQ.24.1.1

Stein, V. (1990). Exploring the Cognition of Reading-to-Write. In L. Flower, V. Stein, J. Ackerman, M. J. Kantz, K. McCormick, \& W. C. Peck (Eds.), Reading to Write. EXploring a Cognitive \& Social Process (pp. 119-143). Oxford University Press.

Wang, P. C., Liu, B. Q., \& Wang, H. (2020). The Role of Task Representation in Completing an Integrated L2 Writing Task: Evidence from Eye-Tracking and Stimulated Recall. Open Journal of Modern Linguistics, 10, 773-784.

https://doi.org/10.4236/ojml.2020.106047

Weigle, S. C., \& Parker, K. (2012). Source Text Borrowing in an Integrated Reading/Writing Assessment. Journal of Second Language Writing, 21, 118-133.

https://doi.org/10.1016/j.jslw.2012.03.004 